\title{
Beam ion instability: Measurement, analysis, and simulation
}

\author{
L. Wang, J. Safranek, Y. Cai, J. Corbett, R. O. Hettel, T. O. Raubenheimer, J. Schmerge, and J. Sebek \\ SLAC, MS 66, 2575 Sand Hill Road, Menlo Park, California 94025, USA
}

(Received 22 May 2013; published 3 October 2013)

\begin{abstract}
A weak vertical coupled-bunch instability with oscillation amplitude of the order of a few $\mu \mathrm{m}$ has been observed in SPEAR3 at nominal vacuum pressure. The instability becomes stronger with increasing neutral gas pressure as observed by turning off vacuum pumps, and becomes weaker when the vertical beam emittance is increased. These observations indicate that the vertical beam motion is driven by ions trapped in the periodic potential of the electron beam. In this paper we present a series of comprehensive beam measurements, impedance-based stability analysis, and numerical simulations of beam-ion interactions in SPEAR3. The effects of vacuum pressure, gas species, beam current, bunch fill pattern, chromaticity, and vertical beam emittance are investigated.
\end{abstract}

DOI: 10.1103/PhysRevSTAB.16.104402

PACS numbers: 29.27.Bd, 29.20.db

\section{INTRODUCTION}

In an electron storage ring, ions generated from electron beam collisions with residual gas molecules can be trapped by the periodic electric potential of the bunched beam. The positively charged ions then interact resonantly with the beam leading to coupled-bunch motion and emittance blowup. For this reason most storage ring light sources use a long bunch train followed by a charge-free gap to avoid multiturn ion trapping. Nevertheless, the ionclearing gap often does not preclude ions from accumulating during one passage of the bunch train resulting in the fast ion instability (FII) predicted by Raubenheimer and Zimmermann [1]. The FII has been observed at the Advanced Light Source and Pohang Light Source by artificially increasing the neutral gas pressure with helium injection into the vacuum chamber, or by turning off vacuum pumps to induce pressure buildup [2-4]. Similarly, as observed in PLS [5], SOLEIL [6], and Shanghai Synchrotron Radiation Facility [7], when the vertical beam emittance is reduced the trapping potential increases and beam-ion instabilities can occur at nominal vacuum pressure.

Although beam-ion instabilities have been qualitatively observed at many accelerator facilities, there is still a need to develop quantitative agreement between measurement, theory, and simulation. Existing theories often include a single damping factor, for instance, Stupakov's [8] investigation of beam size variation around the accelerator and Kim/Ohmi [9] and Wang's [10] formalism of including nonlinear space charge effects. Here we provide a detailed analysis of beam-ion interactions in SPEAR3 including the effect of multiple gas species, realistic beam

Published by the American Physical Society under the terms of the Creative Commons Attribution 3.0 License. Further distribution of this work must maintain attribution to the author(s) and the published article's title, journal citation, and DOI. optics, nonlinear space charge forces, and chromaticity simultaneously. All these factors can provide damping mechanisms which act to suppress growth of beam-ion instabilities. This integrated-physics approach yields good agreement between measurement and calculated beam instability in SPEAR3.

It is important to note some basic characteristics of beam-ion instabilities to correctly observe and interpret the measured data. Ions trapped in the electron beam potential oscillate with a frequency dependent on beam current, beam size around the ring, and bunch fill pattern. The trapped ion oscillation frequency can range from $10-150 \mathrm{MHz}$ in SPEAR3 which corresponds to an ion oscillation period of 100-6 ns or 50-3 rf buckets. The oscillation frequency also varies with charge/mass ratio so a mixture of gas species will broaden the effective ion frequency. Since the ion oscillation period is longer than the $2.1 \mathrm{~ns}$ bunch spacing, ions disturbed by one electron bunch can couple to the trailing bunches leading to coupled-bunch instability with mode number correlated to the ion oscillation frequency. The dependence of mode number on beam current profile and transverse beam size also generates important signatures of the beam-ion instability mechanism.

Different from other types of chamber-impedance driven instabilities, the amplitude of a beam-ion instability typically grows quickly in the linear regime and then saturates at low amplitude, about one beam radius $[13 \mu \mathrm{m}$ rootmean-square (rms) size in our case], due to nonlinear amplitude-dependent space charge effects. Since SPEAR3 has mode-damped cavities and low vacuum chamber impedance, coupled-bunch instabilities driven by resistive wall impedance and high-order cavity modes are normally small or nonexistent. This makes SPEAR3 an ideal machine to isolate and measure beam-ion interactions.

This paper reports on measurement and modeling of beam-ion instabilities in SPEAR3 including single bunch train (FII) and multiple bunch-train instability phenomena. 
Note that the instability characteristics may differ even with the same electron beam conditions due to changes of the vacuum characteristics over time. Most of the measurements were made under nominal vacuum conditions with the exception of one case where the vacuum pressure was artificially increased by turning off ion pumps. The paper is organized with measured data presented in Sec. II, wakefield/impedance analysis in Sec. III, and numerical simulations in Sec. IV.

\section{BEAM-ION MEASUREMENTS}

\section{A. The SPEAR3 light source}

The SPEAR3 storage ring has an 18-cell double bend achromat racetrack magnet lattice with $234 \mathrm{~m}$ circumference and harmonic number 372 . At $3 \mathrm{GeV}$ the natural horizontal emittance is $10 \mathrm{~nm}$. The nominal vertical emittance is $10-15 \mathrm{pm}$ for user operations or as low as $5 \mathrm{pm}$ during minimum coupling tests. The nominal fill pattern consists of six bunch trains containing a total of 280 bunches. Four of the bunch trains have 47 consecutive bunches with bunch-train separations of $30 \mathrm{~ns}$ while the other two contain 46 bunches with bunch-train separations of $32 \mathrm{~ns}$. Under these conditions there are no observable beam-ion instabilities at beam currents up to $500 \mathrm{~mA}$.

Table I lists the main parameters of the SPEAR3 storage ring. The beam-loaded vacuum pressure ranges from 0.1 to 0.5 nTorr depending on position in the storage ring. In general, gas composition of different accelerators can vary. In SPEAR3 the dominant species are $\mathrm{H}_{2}(48 \%), \mathrm{H}_{2} \mathrm{O}$ (21\%), $\mathrm{CO}_{2}(14 \%), \mathrm{CO}(12 \%)$, and $\mathrm{CH}_{4}(5 \%)$. These are average values measured at four vacuum gauges. We will use this gas composition as a baseline throughout the paper.

TABLE I. Typical SPEAR3 machine parameters.

\begin{tabular}{lcc}
\hline \hline Parameter & Symbol/unit & Value \\
\hline Beam energy & $E(\mathrm{GeV})$ & 3 \\
Circumference & $C(\mathrm{~m})$ & 234 \\
Beam current & $I(\mathrm{~mA})$ & $200-500$ \\
Revolution frequency & $f_{0}(\mathrm{MHz})$ & 1.280 \\
rf frequency & $f_{\mathrm{rf}}(\mathrm{MHz})$ & 476.315 \\
Harmonic number & $h$ & 372 \\
Bunch spacing & $S_{b} / c(\mathrm{~ns})$ & 2.1 \\
Bunch number & $M$ & 280 \\
Bunch length & $\sigma_{z}(\mathrm{~mm})$ & 6 \\
Momentum compaction factor & $\alpha$ & $1.6 \times 10^{-3}$ \\
Energy spread & $\sigma_{E}$ & $9.8 \times 10^{-4}$ \\
Damping times & $\tau_{x} / \tau_{y} / \tau_{z}(\mathrm{~ms})$ & $4.0 / 5.3 / 3.2$ \\
Horizontal tune & $\nu_{x}$ & 14.1 \\
Vertical tune & $\nu_{y}$ & 6.18 \\
Horizontal emittance & $\varepsilon_{x}(\mathrm{~nm})$ & 10 \\
Vertical emittance & $\varepsilon_{y}(\mathrm{pm})$ & 14 \\
Vacuum pressure & $P(\mathrm{nTorr})$ & $0.1-0.5$ \\
\hline \hline
\end{tabular}

\section{B. Dependence on vacuum pressure}

The beam-ion instability was first observed at SPEAR3 for $200 \mathrm{~mA}$ beam with single bunch-train fill pattern. Figure 1 shows a section of the beam power spectrum where the high peak signals are revolution harmonics and the lower sidebands to the left are vertical sidebands (due to beam-ion coupling in this case). The modal frequencies of the sidebands agree well with theoretical predictions. No horizontal sidebands were observed. Figure 2 shows the bunch-by-bunch vertical oscillation amplitude along the 280 bunch train as a function of bunch number. A single "probe" bunch with higher beam current can be seen in the charge-free gap. Along the main bunch train the observed bunch oscillation amplitude increases from front to back, but not exactly monotonically, and saturates at $\sim 10 \mu \mathrm{m} \mathrm{rms}$ (about one vertical beam size). Since coupled-bunch instabilities driven by traditional vacuum chamber impedance do not exhibit similar saturation mechanisms the observed beam motion can be attributed to beam-ion interactions.

In order to confirm the beam-ion coupling hypothesis, the vacuum pressure was raised by turning off a series of ion pumps in the ring. Figure 3 shows the resulting growth of the vertical sidebands for a range of different average pressures and $300 \mathrm{~mA}$ total beam current. In this case, the bunch fill pattern included six bunch trains and the frequencies indicated along the horizontal axis represent the location of the vertical low sideband frequencies near the revolution harmonics. Initial operation with all vacuum pumps active produces an average ring pressure of 0.37 nTorr and no vertical beam instability. The instability began to appear as the neutral gas pressure was increased and became stronger at higher pressures. These observations confirmed

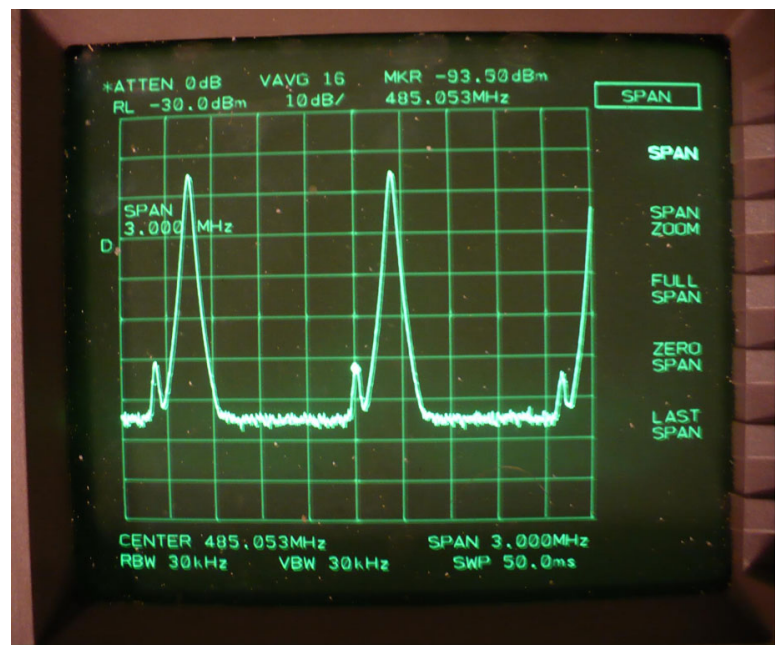

FIG. 1. Beam spectrum at $200 \mathrm{~mA}$ with a single bunch-train fill pattern. The high peaks are the revolution harmonics and the peaks to the left are lower vertical sidebands excited by beam-ion interactions. 


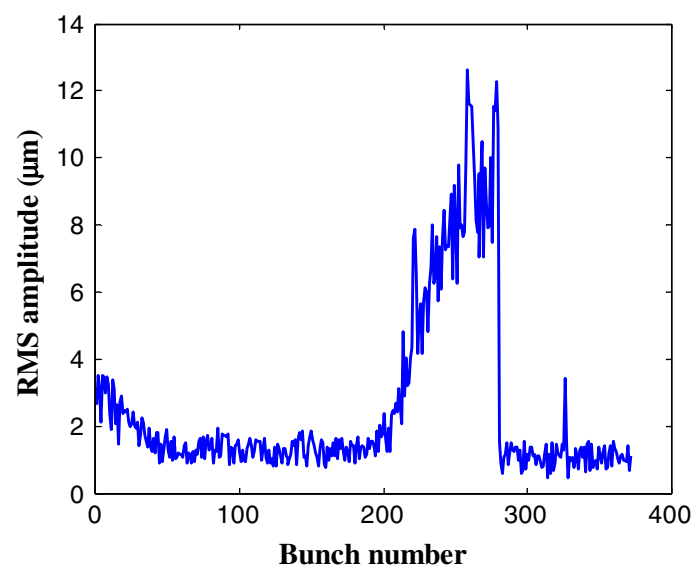

FIG. 2. Bunch-by-bunch vertical oscillation amplitude with a single 280-bunch train. Bunches 1-280 are filled with a total beam current of $200 \mathrm{~mA}$. A single probe bunch is visible in bucket 326.

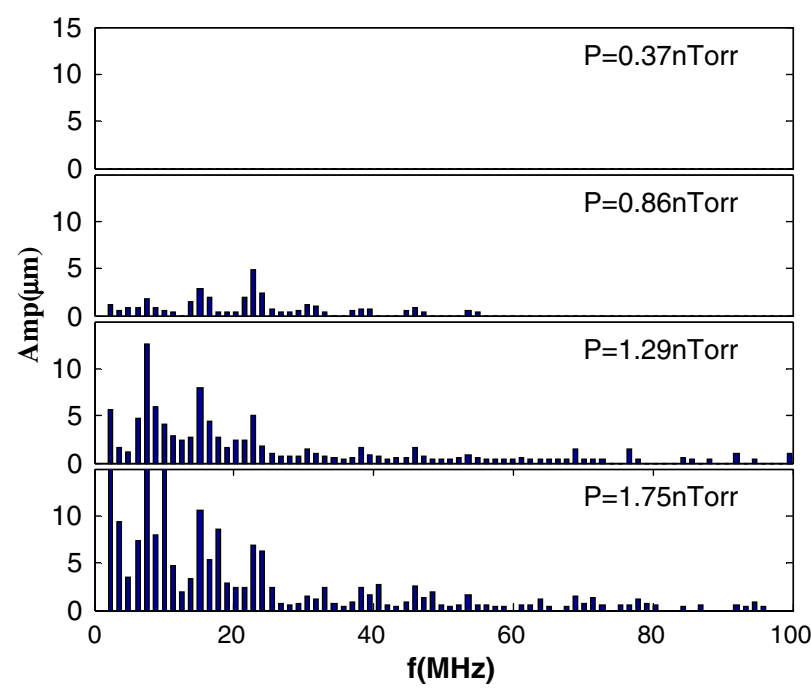

FIG. 3. Vertical lower sideband spectra at different total vacuum pressures. For these measurements the beam consisted of six bunch trains with total bunch number of 280 and beam current $300 \mathrm{~mA}$. No sidebands are visible at the nominal operating pressure of 0.37 nTorr.

that the vertical instability was driven by ions in the vacuum chamber.

As discussed above, the ion oscillation frequency can vary around the accelerator due to changes in the periodic electron beam trapping potential and ion charge/mass ratio. As shown in Fig. 3, the combined effects of variable beam size and complex gas composition result in a broad spectrum of unstable modes with relatively low oscillation amplitudes even at higher vacuum pressure.

\section{Dependence on vertical beam size}

Additional tests were carried out to confirm the effect of vertical emittance on the beam-ion interaction. For these measurements the skew quadrupole magnets were turned off to increase vertical beam size. Figure 4 shows the resulting change in the spectrum of lower vertical sidebands with the skew quadrupole magnets on and off. The vertical beam size is about 2.3 times larger when the quadrupole magnet is turned off. In this case the beam fill pattern consisted of a single bunch train with 280 bunches and a total beam current of $192 \mathrm{~mA}$. With the skew quadrupole magnets off, the maximum frequency of the sidebands decreased from 26.0 to $13.0 \mathrm{MHz}$, and the spectral amplitude dropped as predicted by the theory when considering the change in focusing potential as seen by the trapped ions. This behavior also indirectly confirmed that the beam instability was driven by ions rather than physical vacuum chamber impedance because to first order the resistive wall and geometric impedance do not change with beam size. The observed frequencies also agree with estimated values presented below.

Note that hydrogen ions do not contribute to the beamion instability although it is the dominant gas species in the SPEAR3 vacuum chamber. This can be explained by the small ionization cross section and low molecular mass. We rarely observe beam instabilities at the higher frequencies expected from hydrogen ions even at high beam current. Generally speaking, heavy ions are the primary drivers of beam-ion instabilities due to their large ionization cross section, slower kinetic velocity, and consequently more stable motion in the periodic trapping potential. The simulation shows that the ion volume density exhibits an exponential decay in the charge-free gap with a decay time order of the ion's oscillation period. Therefore, the bunchtrain gap is more effective in reducing the ion density for light ions, which also reduces the effect of light ions. As demonstrated below, heavier ions contribute to larger effective beam-ion impedance due to these effects.

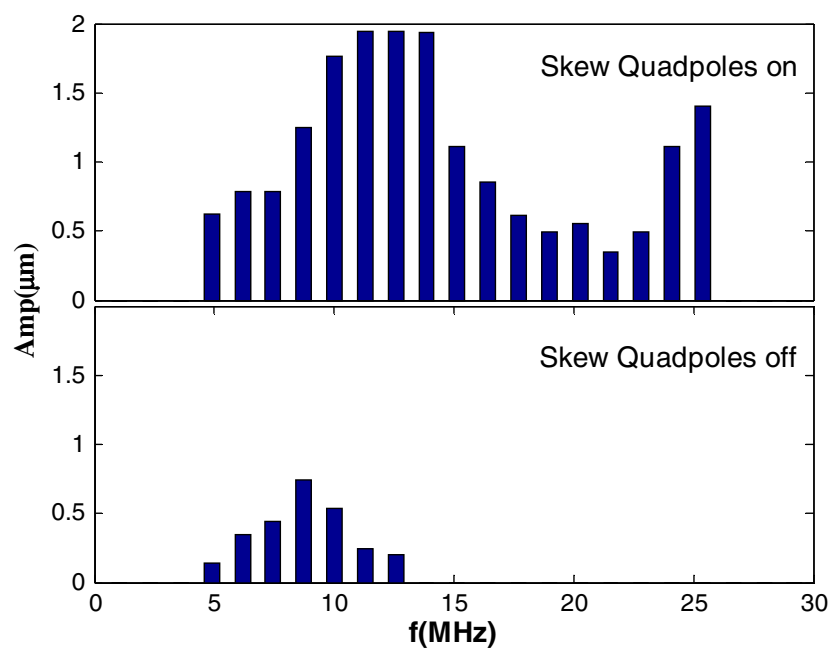

FIG. 4. Lower vertical sideband spectra in a single bunch-train fill pattern with 280 bunches and total beam current of $192 \mathrm{~mA}$ for different beam coupling conditions: skew quadrupole magnets on and off. 


\section{Dependence on total beam current}

Total beam current affects the beam-ion interaction in two ways: the frequencies of the unstable modes increase with the square root of the beam current, and the instability growth rate increases linearly with beam current with the assumption of constant vacuum and long enough bunchtrain gap. Figure 5 shows the lower vertical sideband spectra for various beam currents and again a single bunch train. Clearly there is a tendency toward stronger instability at higher beam currents. Other types of beam instability also have the same character, however in this case the amplitude of the unstable modes remains small, which is a typical feature of beam-ion instabilities. The increase in the unstable modal frequencies with beam current is a characteristic of beam-ion instabilities. Note that the unstable modes at lower frequency always appear when strong instability occurs, for instance at high beam current,

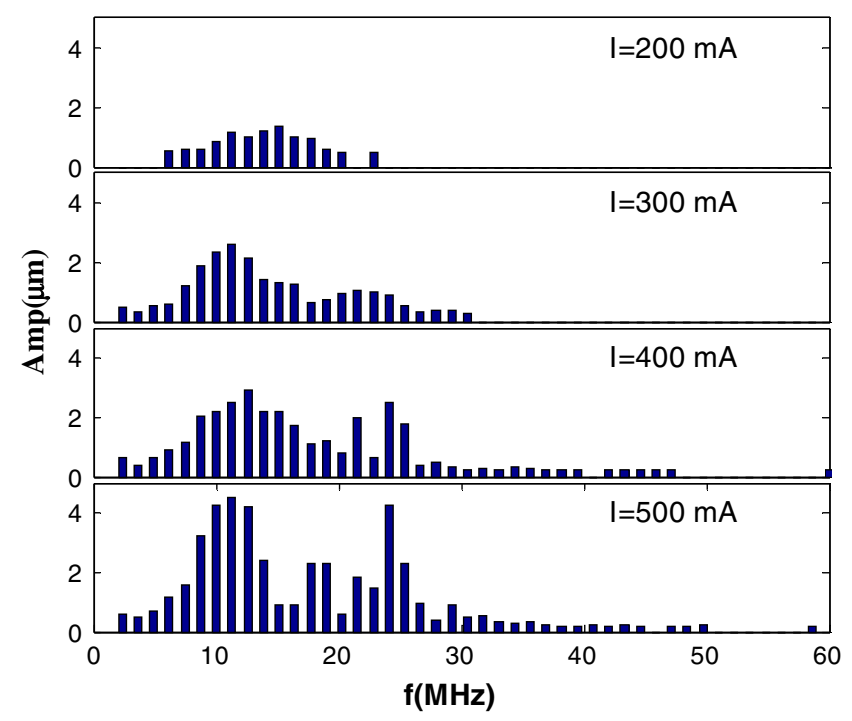

FIG. 5. Vertical lower sideband spectra at different beam currents. The beam has single bunch train with 280 bunches.

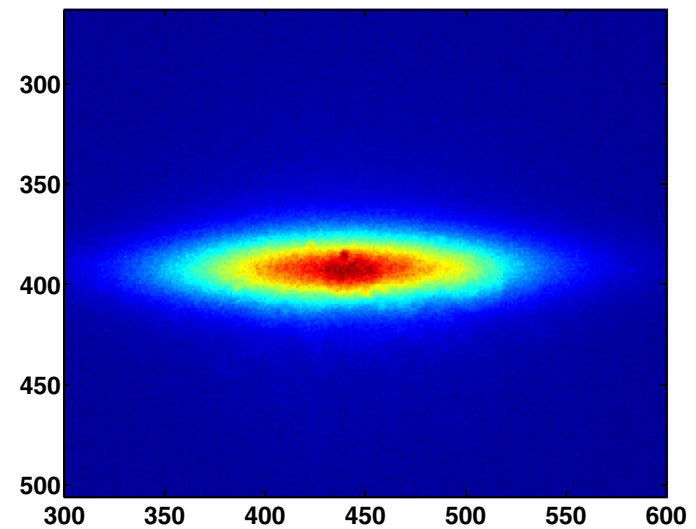

high vacuum pressure, or short bunch-train gap. When the amplitude of the beam instability grows larger than the beam size, the oscillation amplitude enters a nonlinear regime which results in larger frequency spread towards low frequencies and consequently stronger Landau damping of the motion.

\section{E. Dependence on bunch fill pattern}

Beam-ion interactions are particularly sensitive to the bunch fill pattern because ion accumulation depends on the timing structure of effective trapping potential presented by the electron beam. In general, more ions survive a short gap in the bunch train because time of flight in the fieldfree region is reduced. As demonstrated in SPEAR3, the beam-ion instability becomes slightly stronger when the train gap was reduced from 189 to $38 \mathrm{~ns}$ and was much stronger when the gap was reduced to $17 \mathrm{~ns}$. These results suggest that the effect of gap size is nonlinear-a gap of $38 \mathrm{~ns}$ was tolerable but a gap of $17 \mathrm{~ns}$ was too short. For example as shown in Fig. 6, pinhole camera images of the $\mathrm{x}$-ray beam clearly identify ion-induced vertical beam size blowup in the extreme case of no ion-clearing gap.

As a result, a multi-bunch-train fill pattern can help to mitigate beam-ion instabilities by reducing the density of trapped ions near the beam [10]. For example, the beam is unstable with a single bunch train $200 \mathrm{~mA}$ current beam as shown in Fig. 1 but the instability disappears when the single bunch train is divided into two equal bunch trains (not shown here).

Figure 7 shows the lower sideband amplitudes of unstable modes with one, four, and six bunch trains. The beam current is $500 \mathrm{~mA}$ in all cases. Note that there is significant reduction with four bunch trains compared with a single bunch train. However, the rate of reduction decreases beyond four bunch trains. The optimum fill pattern appears to be six bunch trains, which was chosen for the nominal SPEAR3 operating mode. The bunch-train gap for the six-bunch-train fill pattern is 32 ns (15 buckets) which is equal to one wavelength at $31 \mathrm{MHz}$.

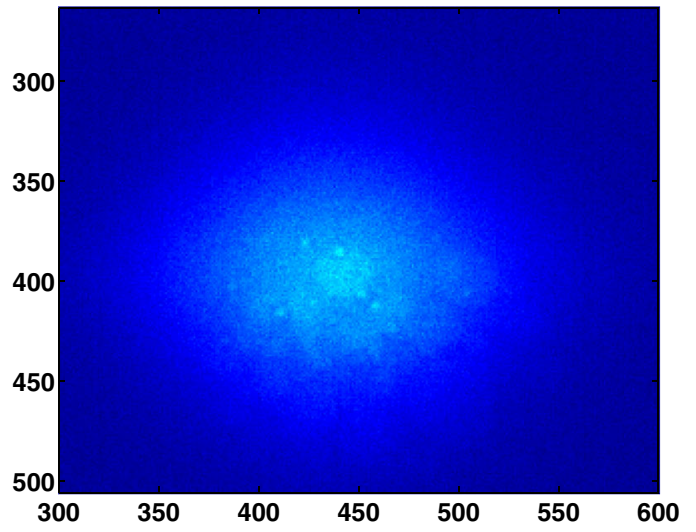

FIG. 6. Beam profile change for $200 \mathrm{~mA}$ single bunch train with $42 \mathrm{~ns}$ gap (left) and with no gap (right). The beam has uniform fill pattern in the no gap case. 


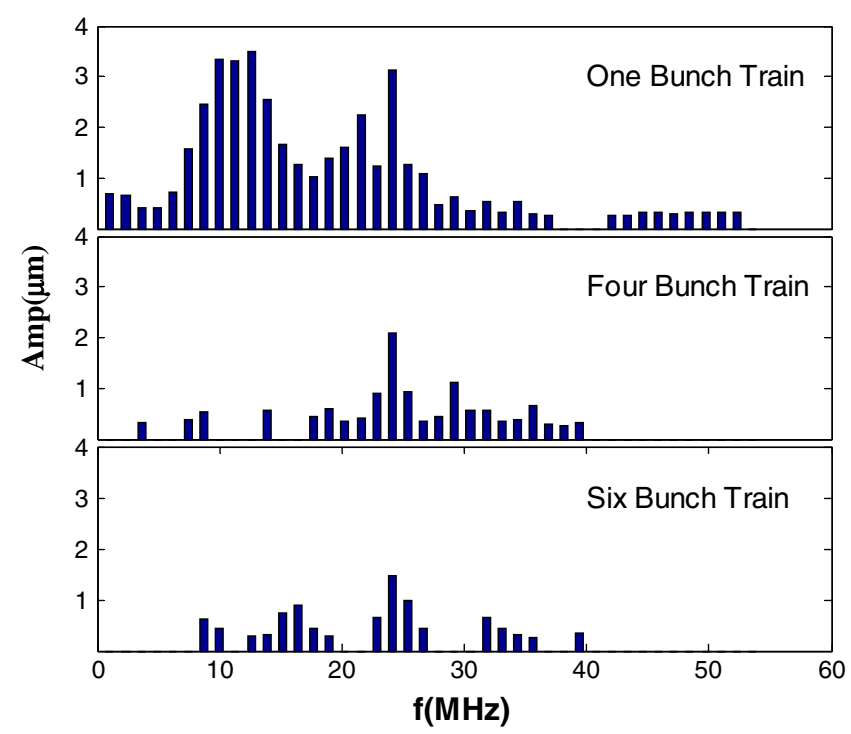

FIG. 7. Spectral amplitude of vertical lower sidebands for different beam fill patterns: one, four, and six bunch trains. The total beam current is $500 \mathrm{~mA}$ with a total bunch number of 280 in all cases. The horizontal and vertical chromaticities are 1.6 and 2.0 , respectively.

\section{F. Dependence on chromaticity}

In addition to using different bunch-train fill patterns, the beam-ion instability can also be controlled by increasing chromaticity. Figure 8 shows the reduction in lower vertical sidebands with higher vertical chromaticity in SPEAR3. In this case, the beam fill pattern was a single 280-bunch train with total beam current of $500 \mathrm{~mA}$ and the horizontal chromaticity was held constant at 2.0. The data

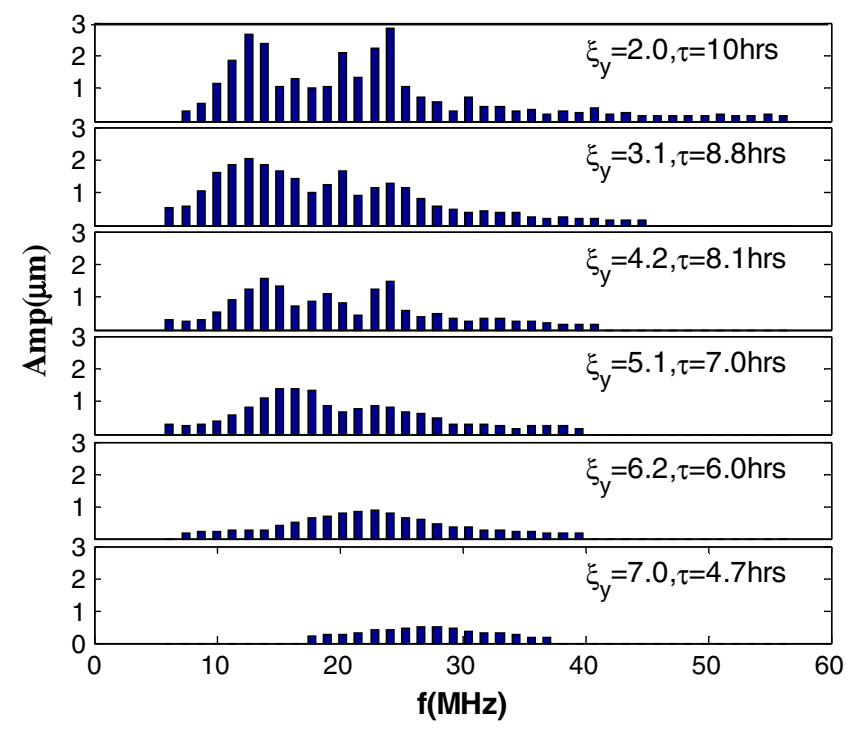

FIG. 8. Spectral amplitudes for lower vertical sidebands with varying vertical chromaticity in SPEAR3. The beam has a single 280-bunch train and a total beam current of $500 \mathrm{~mA}$ in each case. Beam lifetime is also shown on the plots. clearly show the damping effect from increased vertical chromaticity. Note that the additional nonlinearity in the lattice optics at high chromaticity, such as $\xi_{y}=7$ as shown in Fig. 8, decreases the dynamic aperture and therefore drops the beam lifetime from 10 to 4.7 hours and reduces the beam injection rate. SPEAR3 normally operates with top-up injection at 5 min intervals so control of the beamion instability with excessively large chromaticity is impractical.

Based on the bunch fill pattern studies outlined above and the desire to operate with modest chromaticity, SPEAR3 now operates with either four or six bunch trains. Beam-ion instability at $500 \mathrm{~mA}$ with a six-bunch-train pattern is completely suppressed by increasing the vertical chromaticity from 2.0 to 2.6 without the use of bunch-bybunch feedback. Feedback may become necessary for future lower-emittance operation or with more small-gap insertion devices. When on occasion the beam-ion instability does appear (which can happen following a vent of the vacuum chamber), a slightly higher vertical chromaticity can be applied to suppress the instability.

\section{G. Resistive wall instability}

For comparison, we can briefly discuss the resistive wall instability which has been observed in many light sources due to the use of stainless steel vacuum chambers and small aperture insertion devices. In SPEAR3 the vacuum chamber is constructed from oxygen-free high-conductance copper and many of the ID chambers are internally copper plated so there is no resistive wall instability in nominal operating conditions. In order to detect resistive wall instability threshold, we injected a uniform bunch pattern (no gap). In this case, a low frequency instability was observed in the vertical plane. Figure 9 shows the resulting lower sideband spectrum with a total beam current of $500 \mathrm{~mA}$. The beam instability can be attributed to resistive wall impedance because the spectrum contains signature sideband amplitudes inversely proportional to the square root of frequency. Under these conditions the instability could not be passively suppressed even at high vertical chromaticities up to 7.4.

For the single 280-bunch-train beam including an ionclearing gap, we observed only pure beam-ion instability with a vertical chromaticity of 1.4. The resistive wall instability appeared at lower chromaticity of 0.4. The resistive wall instability becomes slightly stronger with multiple bunch-train patterns where the threshold in terms of vertical chromaticity is about $\xi_{y}=0.9$ for two, four, and six bunch trains. These results indicate that the resistive wall instability also depends on the bunch fill pattern and is strongest with a uniform fill pattern. On the other hand, the single bunch-train fill pattern with a gap has slightly weaker resistive wall instability than multiple bunch-train cases. The resistive wall instability in SPEAR3 with nominal multi-bunch-train beam is suppressed by a chromaticity 


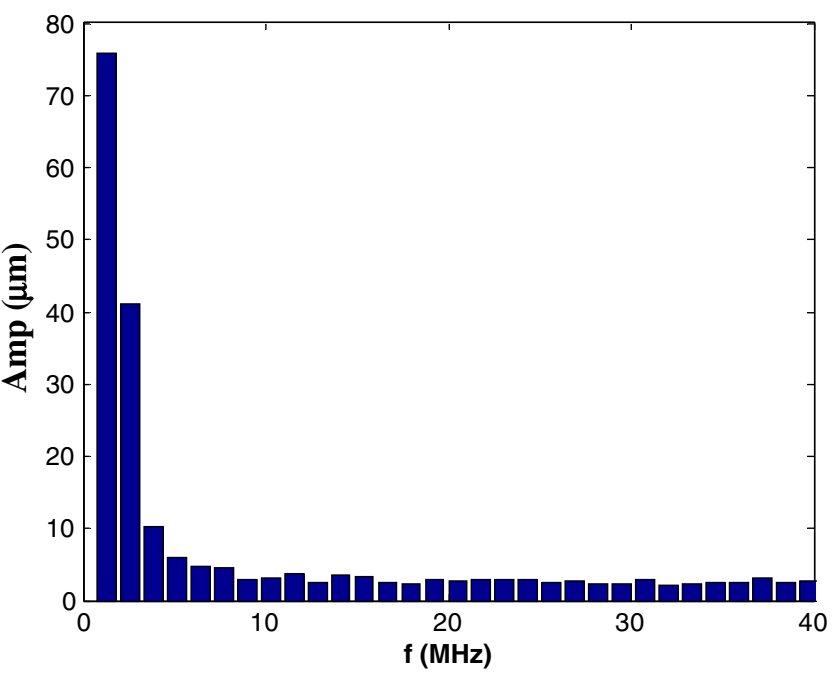

FIG. 9. Spectral amplitude of lower vertical sidebands due to resistive wall instability with a uniform fill pattern at $500 \mathrm{~mA}$ and chromaticity of 2.2 and 5.4 in horizontal and vertical planes, respectively.

about 1.0. A bunch-by-bunch feedback may become necessary when more insertion devices are installed in the future.

\section{THEORETICAL ANALYSIS}

\section{A. Ion-cloud impedance with arbitrary vacuum and variable beam size}

To compare theory with measurements, it is essential to model the beam-ion instability with accurate gas composition vacuum and include variation of beam size around the ring. Existing beam-ion instability theories do not include both effects. Our analysis can model multiple gas species, realistic beam optics, nonlinear space charge forces, and chromaticity simultaneously. Therefore, it can be directly used to compare with beam measurements.

To begin, we model the beam-ion instability as a two-stream beam instability. In this case positive ions are trapped by the negatively charged electron beam. The electric field of a bi-Gaussian distribution of particles in the linear regime (within one beam size) has the following form:

$$
E_{x, y}=\frac{\lambda}{2 \pi \varepsilon_{0} \sigma_{x, y}\left(\sigma_{x}+\sigma_{y}\right)}(x, y),
$$

where $\lambda$ is the line density of the charge and $\varepsilon_{0}$ is the vacuum permittivity. The ion-cloud distribution is not exactly Gaussian but the electric field of the ion cloud can be assumed to closely approximate that of a Gaussian distribution with $\sigma_{i}=\sigma_{e} / \sqrt{2}$ [10].

In the linear regime, the coupling force between the electron beam and ion cloud can be modeled using the wakefield formalism [10]. Here, when an electron bunch with transverse displacement $\Delta y_{e}$ passes through the ion cloud, the ion cloud begins to oscillate with frequency of $\omega_{i}$ and amplitude of $\hat{y}_{i}$. The oscillating ion cloud then deflects trailing electron bunches which further amplifies the ion motion. When the offset $\Delta y_{e}$ is smaller than the beam size and the wavelength of the ion-cloud oscillation is much longer than the bunch spacing, the motion of the ion cloud can be expressed as

$$
y_{i}(t)=\hat{y}_{i} \sin \omega_{i} t=\frac{N_{e} r_{p} c}{\omega_{i, y} A} \frac{2}{\sum_{y}\left(\sum_{x}+\sum_{y}\right)} \Delta y_{e} \sin \omega_{i} t,
$$

where $c$ is the speed of light, $r_{p}$ is the classical radius of the proton, $A$ is the mass number of the ion, $N_{e}$ is electron bunch population, and $\sum_{x, y}$ is an effective cross-sectional area which depends on the transverse size of electron bunch and ion cloud. If electron bunches and the ion cloud both have Gaussian distributions with rms size $\sigma_{e}$ and $\sigma_{i}$, we get

$$
\sum_{x}=\sqrt{\sigma_{e, x}^{2}+\sigma_{i, x}^{2}} \text { and } \sum_{y}=\sqrt{\sigma_{e, y}^{2}+\sigma_{i, y}^{2}} .
$$

Then it is straightforward to calculate the kick of the oscillating ion cloud on subsequent electron bunches. The wakefield is derived from the ratio of the kick relative to the initial offset as

$$
W_{y}(s)=\frac{\gamma}{N_{e} r_{e}} \frac{\Delta \bar{y}_{e}^{\prime}(s)}{\Delta y_{e}},
$$

where $r_{e}$ is the classical radius of electron and $\gamma$ is the relativistic factor. In the linear regime, the kick amplitude is proportional to the offset so the wakefield is independent of offset as expected. The wakefield of an ion cloud with a constant beam size can be further generalized to include the nonlinear field as [10]

$$
W_{y}(s)=\hat{W}_{y} e^{-\left(\omega_{i} s / 2 Q_{0} c\right)} \sin \left(\frac{\omega_{i} s}{c}\right),
$$

here the amplitude of the wake is given by

$$
\hat{W}_{y}=N_{i}\left(\frac{r_{p} S_{b}}{A N_{e}}\right)^{1 / 2}\left[\frac{4}{3} \frac{1}{\sigma_{y}\left(\sigma_{y}+\sigma_{x}\right)}\right]^{3 / 2},
$$

where $N_{i}$ is the number of ions, $S_{b}$ is bunch spacing, and $\sigma_{x, y}$ is the transverse rms beam size of the electron bunches. $Q_{0}$ is an effective quality factor due to the nonlinear space charge field, which depends on the distribution of the particles. For a Gaussian distributed beam, $Q_{0} \sim 9$ [10]. It is important to note that the nonlinearity has only a weak dependence on the vertical beam emittance when the horizontal emittance is fixed. For instance, the $Q_{0}$ value changes from 8.63 to 9.74 when the vertical emittance is increased from 1 to $8 \mathrm{~nm}$ in our simulations. In the linear regime, the coherent ion oscillation frequency is 


$$
\omega_{i, y}=2 \pi f_{i, y} \approx c\left(\frac{4 N_{e} r_{p}}{3 A S_{b}\left(\sigma_{x}+\sigma_{y}\right) \sigma_{y}}\right)^{1 / 2} .
$$

For a flat beam, as seen in most electron rings, the vertical wake function is larger than the horizontal wake function resulting in a stronger instability in the vertical plane. This explains the reason we predominantly observe the vertical instability in SPEAR3. In the rest of this paper we discuss only vertical beam-ion instabilities and omit the subscript $y$ for simplicity.

In general, the beam size and vacuum pressure vary around the ring. The total wakefield from the ions is therefore calculated by integrating around the whole ring [10],

$$
W_{\text {ring }}(s)=\int_{0}^{C} \frac{4}{3} \frac{\omega_{i, y}(z)}{c} \frac{\lambda_{i}(z) S_{b}}{N_{e}} \frac{1}{\sigma_{y}(z)\left[\sigma_{y}(z)+\sigma_{x}(z)\right]} e^{-\left[\omega_{i}(z) s / 2 Q_{0} c\right]} \sin \left(\frac{\omega_{i}(z) s}{c}\right) d z,
$$

where $\lambda_{i}$ is the ion line density. The volume density of the ion cloud increases linearly along the bunch train and exponentially decays during the bunch-train gap with a decay time of the order of the ion oscillation period [10]. Equation (8) can be used to calculate the wake function with arbitrary beam fill pattern if it is assumed that the bunch spacing is much shorter than the ion oscillation period. This condition should be checked at each location and the integral should be done only at the locations where the assumption is true. For instance, where there is only a very small betatron function in the wiggler section of the International Linear Collider (ILC) damping ring, the light ions species cannot be trapped along the bunch train. The strong space charge force of the electron bunch "over-kicks" ions and drives them out of the beam.

The total wake function with multiple gas species can be calculated from Eq. (8) by linearly superimposing the wake function of different ion species. As a result, Eq. (8) can yield the total wake function of ions around the ring with arbitrary beam optics and gas composition, including nonlinear space charge effects. It should be pointed out that it is essential to calculate the wake function using Eq. (8) —as is shown later the wake function can be inaccurate if estimated assuming constant beam size around the ring.

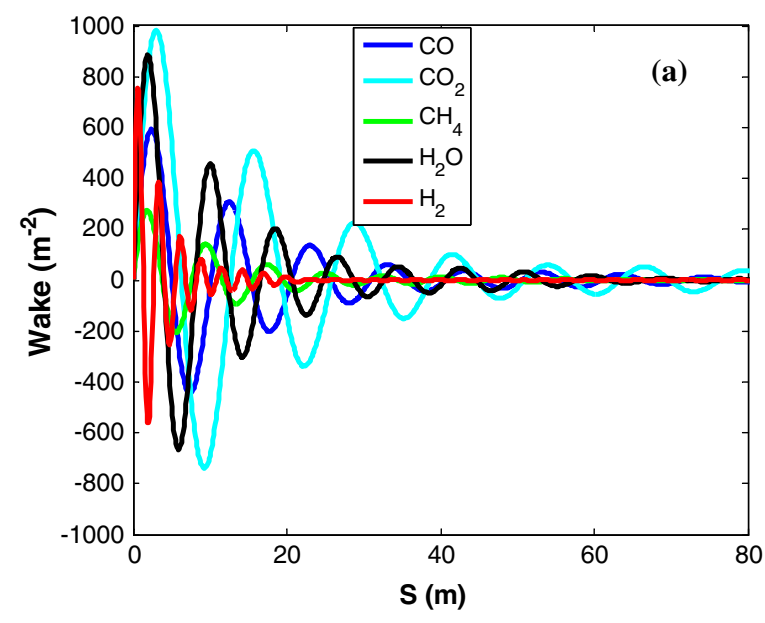

In many cases, it is advantageous to analyze beam instability problems in the frequency domain. The impedance due to the ion cloud integrated around the ring can be obtained by Fourier transform of the wake function as

$$
Z_{\perp}(\omega)=i \int_{-\infty}^{\infty} \frac{d s}{c} W_{\text {ring }}(s) e^{i \omega s / c} .
$$

Time and frequency domain plots of the ion wake function and impedance in SPEAR3 including all effects (nonlinear space charge force, beam optics, and multiple gas species) for a typical gas composition and total gas pressure of 0.37 nTorr are shown in Fig. 10. In this case, the beam fill pattern was six bunch trains with a total beam current of $500 \mathrm{~mA}$. The ion density seen by different bunches varies somewhat because of the nonuniform fill pattern. The wake function was calculated using an average ion density seen by all bunches.

The benchmark against simulation shows the average ion density method is a good approximation with a sufficient number of bunch trains. Similarly, the growth rate of the single bunch-train instability [1] is exactly proportional to the average ion density (or wakefield) along the bunch train. In this case, both the instability growth rate and the wake of ion cloud increase linearly along the bunch train.

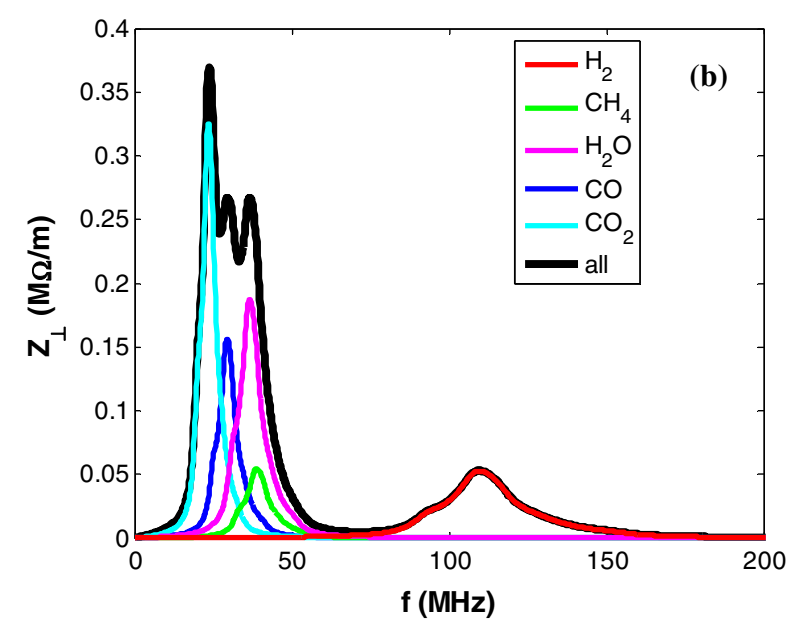

FIG. 10. (a) Vertical wake functions, and (b) real part of impedance due to different ion species in SPEAR3 with total pressure of 0.37 nTorr. The beam fill pattern consists of six bunch trains, total bunch number of 280 and a total beam current of $500 \mathrm{~mA}$. 
This may explain why the average wake model works. The calculated wake function and impedance induced by different ion species shown in Fig. 10 provide further insight into the beam-ion interaction physics. In each case a quality factor of $Q_{0}=9$ was used to model the effect of nonlinear space charge. The wake function from $\mathrm{CO}_{2}$ ions has the largest amplitude due to its large ionization cross section and large mass (low diffusion rate). $\mathrm{H}_{2}$ ions also have large wake function due to the high partial pressure of hydrogen although its ionization cross section is small. However, the frequency of $\mathrm{H}_{2}$ is large so that its effect on instability is small. Clearly each wake function has a damped-resonant oscillation form with wavelength longer than the bunch spacing thus providing a multibunch coupling mechanism.

Through integration, the impedance spectrum for each gas species depends on the variation of the beam size around the ring. The net impedance at each frequency results from contributions of the ions at different locations around the ring taking into account variations in electron beam size. The computed single-gas impedance spectrum therefore captures the frequency-dependent effect of variations in beam size. For instance, the impedance due to single gas species in the ILC damping ring has three peaks which represents the contributions of the ions from straight, arc, and wiggler sections, respectively [11]. Note that the relative frequency spread $\Delta \omega / \omega$ of the impedance induced by each ion species is approximately the same because of the cancellation of the mass factor in the numerator and denominator. It purely represents the effect of variations in beam size. As shown later, it can be represented by a $Q$ factor. In a ring with large variations in beam size, such as an ILC damping ring, a larger relative frequency spread in the ion impedance leads to an increased damping of the beam-ion instability.

A multispecies gas composition also contributes to spread in the effective ion frequency. As shown in Fig. 10(b), the overlap of the frequencies from different types of ions increases frequency spread across the impedance spectrum which tends to mitigate the beam-ion interaction. Note that the frequency of $\mathrm{H}_{2}$ ions is well separated from other ion species. The frequencies of $\mathrm{CH}_{4}, \mathrm{H}_{2} \mathrm{O}, \mathrm{CO}$, and $\mathrm{CO}_{2}$ ions overlap strongly in the range from 20 to $60 \mathrm{MHz}$ making it difficult to distinguish the effect of different ion species experimentally.

\section{B. Instabilities driven by multiple gas species}

When the beam is evenly filled around the ring, the exponential growth rate of the coupled-bunch instability for mode $y_{j}^{\mu} \propto e^{2 \pi \mu j / n_{b}}$ is given by the imaginary component of the coherent frequency shift with mode number $\mu$ [12],

$\Omega_{\mu}-\omega_{\beta}=i \frac{N_{e} M r_{e} c}{2 \gamma T_{0}^{2} \omega_{\beta}} \sum_{p=-\infty}^{\infty} Z_{\perp}\left[\left(p M+\nu_{y}+\mu\right) \omega_{0}+m \omega_{s}\right]$

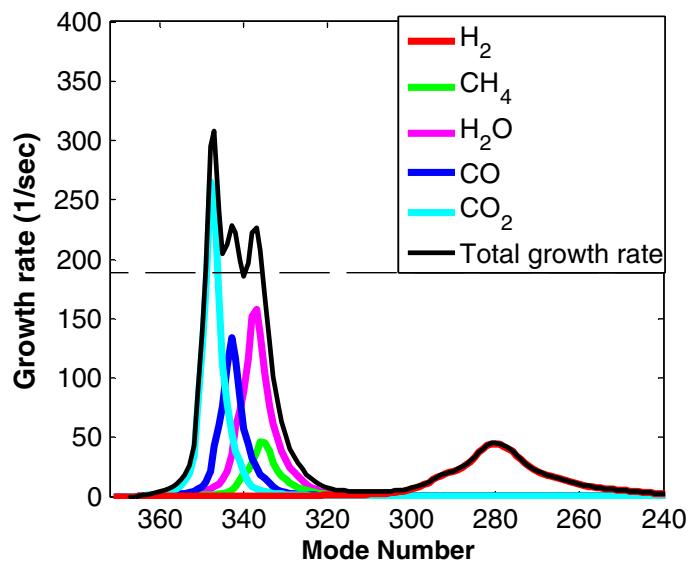

FIG. 11. Growth rates of unstable modes driven by various ion species. The total vacuum pressure is 0.37 nTorr and the beam current is $500 \mathrm{~mA}$ with the six-bunch-train beam fill pattern in SPEAR3. The dashed line shows the radiation damping rate. Chromaticity is zero in this case.

Here $T_{0}$ and $\omega_{0}$ are the revolution period and frequency, $\nu_{y}$ is the betatron tune, $M$ is the bunch number, $\omega_{\beta}$ and $\omega_{s}$ are the betatron and synchrotron frequency. The real part of the frequency shift gives a tune shift and the imaginary part gives the exponential growth rate of the coupled-bunch beam-ion instability. Growth time is the inverse of growth rate. A multi-bunchtrain beam fill pattern with short charge-free gaps is an effective way to reduce the ion impedance and therefore mitigate the beam-ion instability. We will use Eq. (10) to estimate the beamion instability growth rate of multi-bunch-train beams with the approximation of a uniformly distributed bunches.

Figure 11 shows the growth rate of each unstable mode driven by different ion species based on the impedances presented in Fig. 10(b). The different contribution from each ion type is clearly shown in the plot. $\mathrm{CO}_{2}, \mathrm{CO}$, and $\mathrm{H}_{2} \mathrm{O}$ contribute to the most unstable modes. The actual instability is weak when considering the radiation damping rate as shown by the dashed line in the plot. The unstable modes driven by hydrogen ions (near mode number 280) are completely radiation damped. Analysis of separate gas species provides a convenient way to study the effect of realistic vacuum conditions and understand the contribution from each ion species. For instance, the effect of $\mathrm{CO}$, $\mathrm{CO}_{2}$, and $\mathrm{H}_{2} \mathrm{O}$ partially add together to contribute a broad mode spectrum with multiple spikes which agrees with measurement. A single gas model cannot explain multiple spikes in the spectrum. In the ILC damping ring [11], even $\mathrm{H}_{2}$ ions can provide damping to unstable modes. It is therefore important to analyze and simulate the beam-ion instability with a realistic gas composition for each accelerator.

\section{Bunch-train effects}

Several mechanisms to suppress beam-ion instability with a multiple bunch-train fill pattern are discussed in 


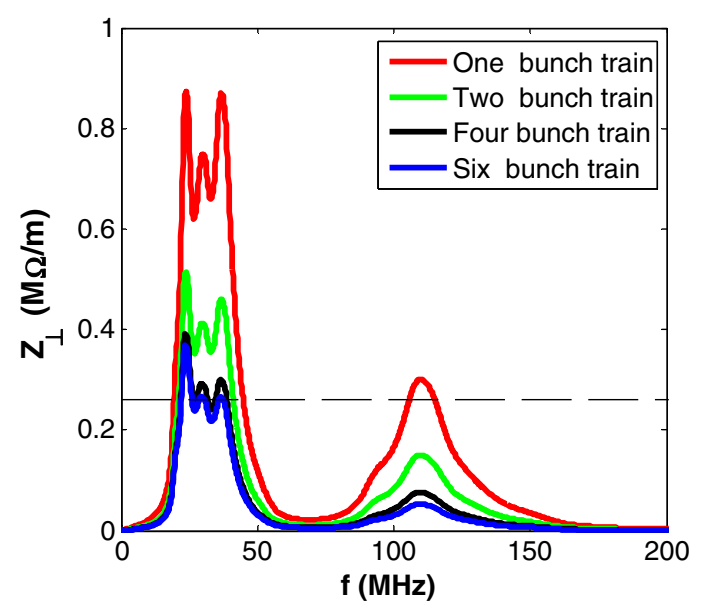

FIG. 12. Calculated of ion-cloud impedance for different beam fill patterns in SPEAR3. The beam current is $500 \mathrm{~mA}$ and the total vacuum pressure is 0.37 nTorr in all cases. The dashed line shows the radiation damping instability threshold with a vertical damping time of $\tau_{y}=5.3 \mathrm{~ms}$. The corresponding instability growth rate with a six bunch train is shown in Fig. 11.

Ref. [10]. Roughly speaking, a multiple bunch-train beam fill pattern can reduce the ion density near the beam and therefore the net ion-induced impedance. Typically, when the bunch-train gap is much longer than the ion oscillation period for a given gas species all ions of that type are cleared. The optimum bunch-train gap is one or two ion oscillation periods to minimize ion trapping while attempting to accommodate as many bunches as possible.

Figure 12 shows the calculated ion-cloud impedance for different beam fill patterns in SPEAR3. There is clearly a reduction in impedance using multiple bunch-train fill patterns. The four and six bunch trains have similar impedance, which agrees with the measurement and simulations shown below. The instability growth rate with the six bunch train was shown in Fig. 11, including radiation damping. The unstable modes driven by $\mathrm{H}_{2}$ ions are very weak for the single bunch train and are completely radiation damped when a multiple bunch train is used. This explains why we do not observe the unstable modes driven by $\mathrm{H}_{2}$ ions in SPEAR3. In general, the frequency range of the calculated impedances shown in Fig. 12 also agrees with the measurement.

\section{Effects of bunched beam and chromaticity}

The above analyses were carried out using a single particle model. For a bunched beam, the beam-ion interaction force technically depends on the product of the impedance and the bunching factor in the frequency domain. The effective impedance seen by a bunched beam is given by

$$
Z_{\perp}^{\mathrm{eff}}=\sum_{p=-\infty}^{\infty} Z_{\perp}(\omega) e^{-\left(\omega-\omega_{\xi}\right)^{2} \sigma_{z}^{2} / c^{2}},
$$

where $Z_{\perp}(\omega)$ is the single particle impedance, $\omega_{\xi}=$ $\omega_{0} \xi / \eta$ is the chromatic frequency shift due to chromaticity $\xi=\Delta Q /(\Delta p / p), \sigma_{z}$ is the rms bunch length, $\eta$ is the slippage factor $\eta=\alpha-1 / \gamma^{2}$, and $\alpha$ is the storage ring momentum compaction factor. Because of the low frequency content of the ion-cloud impedance, the effective impedance seen by a bunched beam at zero chromaticity is almost the same as that of the single particle calculation. As a result, the single particle derivations discussed above are also accurate for a bunched beam. However, nonzero chromaticity can induce a frequency shift which reduces the effective impedance seen by the beam. The impedance reduction depends on both bunch length and the chromatic frequency. The effect of chromatic frequency shift is more pronounced for machines with low momentum compaction factor and/or long bunch length.

For the nominal operation of SPEAR3, both the horizontal and vertical normalized chromaticities are $\sim 2.0$. If the beam-ion instability appears, a slightly higher vertical chromaticity can be used to suppress the instability. Viewed as a function of coupled-bunch mode number, the effect of chromaticity is clearly seen in Fig. 13. For both single bunch-train and multiple bunch-train beams, any weak instability driven by hydrogen ions (mode number 280) can be completely damped by a combination of chromaticity and radiation damping.

According to calculation, with a six-bunch-train beam fill pattern, a chromaticity of 4.0 suppresses all unstable modes except those driven by $\mathrm{CO}_{2}$ ions. Based on measurement, a chromaticity of 2.6 damps the most unstable mode. The difference indicates that either the real total pressure is below 0.375 nTorr or the actual $\mathrm{CO}_{2}$ partial pressure in the vacuum is less that $14 \%$. Nevertheless, the analysis is very close to the measurements even with the

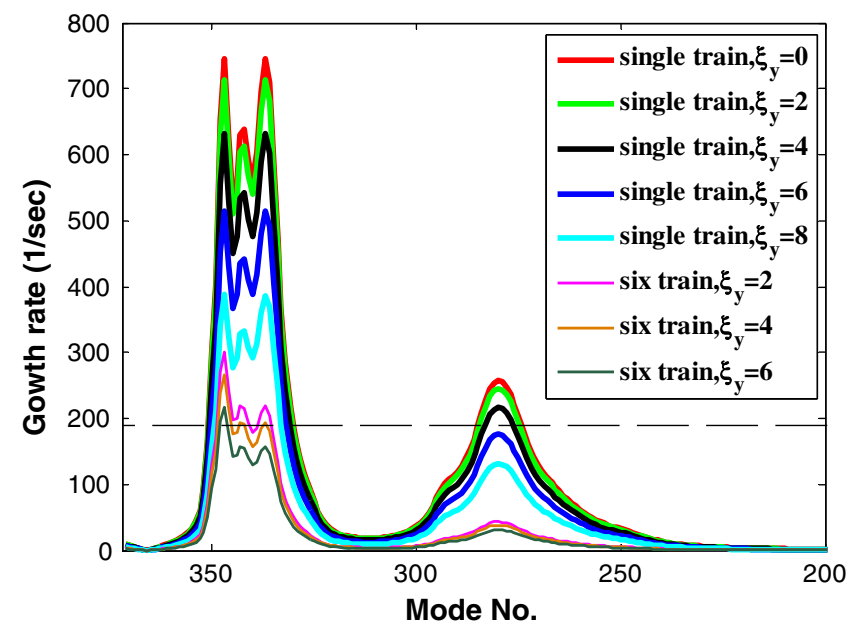

FIG. 13. Calculated growth rates of the beam-ion instability in SPEAR3 for $500 \mathrm{~mA}$ beam current and 280 bunches in a single bunch train and six-bunch-train fill pattern at different chromaticity values. A total average vacuum pressure of 0.375 nTorr is used. The dashed line shows the radiation damping rate. 
uncertainty in gas composition. With $300 \mathrm{~mA}$ beam current and vertical chromaticity of 2.0 , there is no measurable instability which also agrees with analysis.

\section{E. Vertical beam size effects}

As expected, the distribution of unstable modes is closely related to the beam-ion impedance. To see this more clearly, we take the Fourier transform of the resonance-type wake function in Eq. (5) to yield the frequency-dependent impedance,

$$
\begin{aligned}
Z_{\mathrm{ion}}(\omega) & =\frac{\hat{W}_{t}}{\omega} \kappa \frac{Q}{1+i Q\left(\frac{\omega_{i}}{\omega} \frac{1}{\kappa}-\frac{\omega}{\omega_{i}} \kappa\right)} \\
& \approx \frac{\hat{W}_{t}}{\omega} \frac{Q}{1+i Q\left(\frac{\omega_{i}}{\omega}-\frac{\omega}{\omega_{i}}\right)},
\end{aligned}
$$

where $\hat{W}_{t}$ and $Q$ are the amplitude and quality factor, respectively, obtained by fitting the integrated wake function of Eq. (8) to the resonance model in Eq. (5). With $Q \geq 4$, the factor $\kappa=\sqrt{1-(1 / 2 Q)^{2}} \approx 1$. At the resonance frequency, $\omega=\omega_{i}$, the real part of the impedance can be simplified to

$$
\begin{aligned}
& \operatorname{Re}\left\{\sum_{p=-\infty}^{\infty} Z_{\text {ion }}\left[\left(p M+\nu_{y}+\mu\right) \omega_{0}\right]\right\}=\operatorname{Re}\left(Z_{\text {ion }}\left(\omega_{i}\right)\right) \\
& \approx \frac{\hat{W}_{t}}{\omega_{i}} Q=\frac{4}{3} Q \frac{N_{i} S_{b}}{N_{e}} \frac{1}{\sigma_{y}\left(\sigma_{y}+\sigma_{x}\right)} .
\end{aligned}
$$

At SPEAR3, the beam is relatively flat $\sigma_{x} \gg \sigma_{y}$, so the impedance at resonance frequency is inversely proportional to the product of horizontal and vertical beam size.

Vertical beam size (emittance) can affect the ion impedance in several ways for a given bunch current and bunch fill pattern. First, the maximum impedance is roughly proportional to $1 / \sigma_{y}$ when the coupling is less than a few percent and the number of ions is fixed. The problem is complicated, however, by the fact that at fixed current the number of trapped ions depends on beam size as well as ion species. More ions can be trapped with a larger emittance and heavier ions are more stable and have more chance to survive a bunch-train gap. These two factors make heavier ions more important at large vertical beam size. Therefore, as shown in Fig. 14 for different coupling cases, the impedance reduction with large emittance is smaller for heavier ions. For these two cases the ratio of the coupling is 10 and the impedance of light hydrogen ions is reduced by a factor about square root of 10 . The impedance reduction is smaller for heavy ions such as $\mathrm{CO}_{2}$. At large emittance there is less frequency spread and the impedance spectrum shifts to lower frequency. This tendency is shown in Fig. 14 as well as Fig. 4.

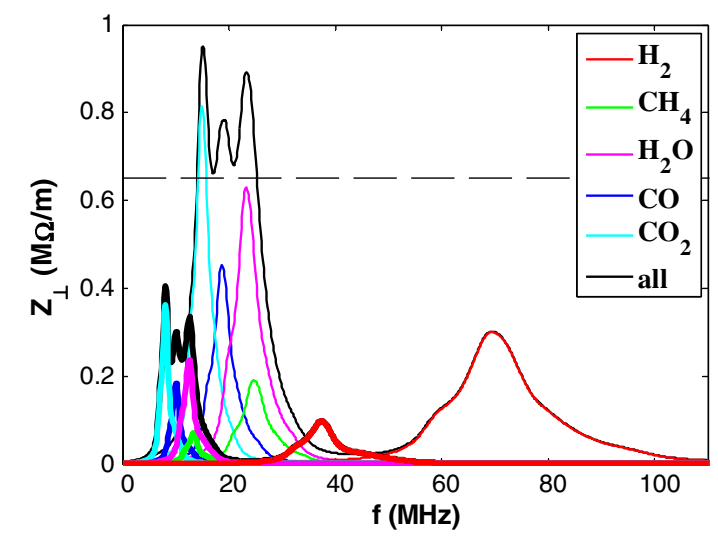

FIG. 14. Ion-cloud impedance with emittance coupling of $0.14 \%$ (thin lines) and $1.4 \%$ (thick lines). In each case, the beam has single 280-bunch train with a total beam current of $200 \mathrm{~mA}$. The total vacuum pressure is 0.37 nTorr in all cases. The dashed line shows the instability threshold due to the radiation damping. There is no instability with an $x-y$ emittance coupling of $1.4 \%$.

\section{F. Beam current effects}

As shown in Eq. (10), for a given fill pattern, the instability growth rate is proportional to the beam current and ion impedance. Interestingly, the ion impedance itself does not increase with beam current, and even slightly decreases with total beam current even though the number of ions goes up. The reason for the net reduction in ion impedance is that the ion frequency also increases with total beam current. The peak impedance is inversely proportional to the ion frequency which reduces the overall impedance at high beam current. As demonstrated in Eq. (13), the ion impedance is roughly proportional to $N_{i} / N_{e}$. For a single bunch train with long gaps, this ratio is approximately constant and the ion impedance therefore does not change with beam current as long as the ions are trapped along the bunch train $\left(\omega_{i} S_{b} / c \ll 1\right)$. For the multi-bunch-train case, the ratio of $N_{i} / N_{e}$ becomes smaller at high beam current because the ions have less chance to survive bunch-train gaps due to the stronger force overfocusing ions [10].

Figure 15 shows the dependence of the ion-cloud impedance on beam current for the one- and six-bunch-train fill patterns. There is a larger frequency spread for a high beam current. The amplitude of the impedance has only a weak dependence on beam current for the single bunch train. With the six bunch trains the impedance decreases with beam current, especially at low frequencies. In this case the bunch-train gap is not long enough to completely clear the ions and the clearing is more effective for high beam current. As a result, the dependence of instability growth rate on total beam current is linear for a single bunch train and slower than linear for multiple bunch trains at a given vacuum pressure. 


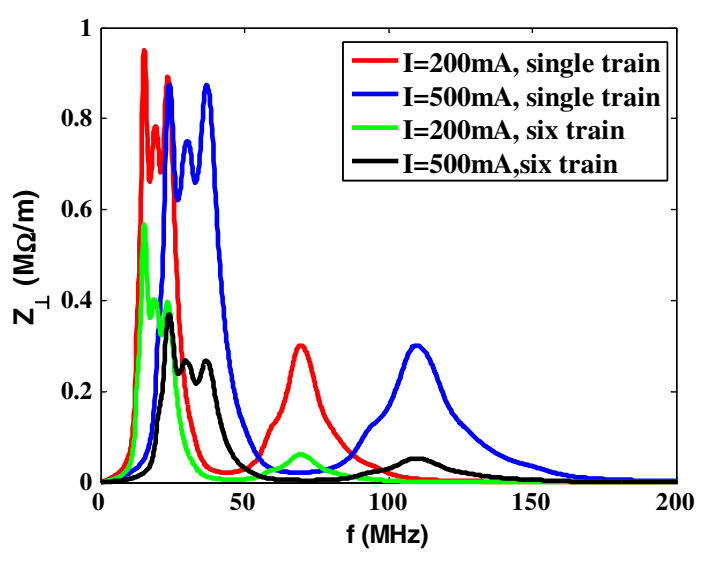

FIG. 15. Dependence of ion impedance on beam current for single- and six-bunch-train fill pattern.

\section{G. Effect of nonlinear space charge forces and beam size variation}

Both the nonlinear space charge force and beam size variation can work to damp beam-ion instabilities. It is therefore interesting to investigate these damping mechanisms independently. The comparison of the effects of nonlinear space charge force and beam size variations is shown in Fig. 16. Three cases are compared: (1) nonlinear space charge forces only; (2) beam optics effect only; (3) both effects. The nonlinear space charge force is turned on and off by setting a low $Q_{0}$ value of 9 and a high $Q_{0}$ value of 200. The beam size variation effect is included or excluded by using a varying or constant beam size, respectively. In the case of constant beam size an average betatron function value is used to calculate beam size.

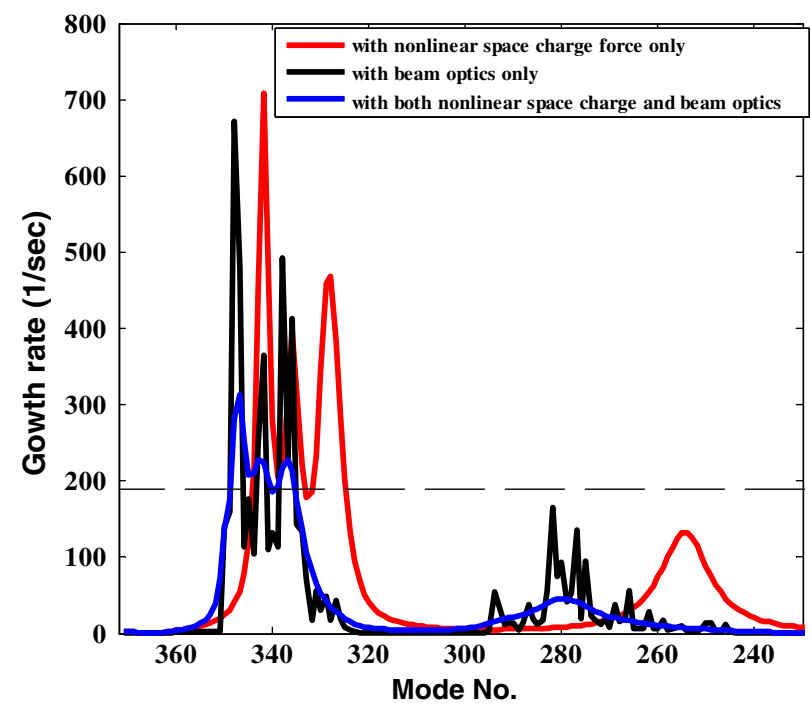

FIG. 16. Beam-ion instability growth rate in SPEAR3 due to different effects: nonlinear space charge effects only; variable beam size effects only and both nonlinear space charge force and beam size effects.
Figure 16 shows that the nonlinear space charge force and variable beam size have a similar damping effect on beam-ion instabilities in SPEAR3. The instability growth rate is reduced about a factor 2 when both effects are included.

It is important to quantify the effect of the variation in beam size which can be characterized by the ion frequency spread [8] and $Q$ [10], respectively. However, it is not clear how to accurately calculate the effect for a given set of variations in beam size around the ring because the distribution of the ion frequencies is usually far from a standard Gaussian distribution. The quality factor $Q$ in the traditional frequency domain analysis is defined as

$$
Q=\frac{\omega_{0}}{\Delta \omega_{0}},
$$

where $\Delta \omega_{0}$ is the full width at half maximum. For instance, if we calculate relative frequency spread in SPEAR3 from the above equation, it yields a $Q$ about 1 . This value is small compared with results shown in Fig. 16 and the more accurate estimate below.

Alternatively, one may approximate the quality factor $Q$ due to beam size variations as the ratio of average frequency around the ring $\bar{\omega}$ to the standard derivation $\sigma_{\omega}$ in frequencies

$$
Q_{\mathrm{optics}} \approx \frac{\bar{\omega}}{\sigma_{\omega}} .
$$

For example, using this technique the estimated $Q$ factor of $\mathrm{CO}$ ions in Fig. 10 is 9.86. This is a good approximation and is close to the more accurate method below.

For a ring with small variations in beam size, like SPEAR3, the wake function due to a single ion species evaluated around the ring still maintains the dampedresonant characteristic shown in Fig. 10(a). In this case, the wake function can be written

$$
\begin{aligned}
W_{\text {ring }}(s) & =W_{0} e^{-\left(\omega_{r} s / 2 Q_{\text {ring }} c\right)} \sin \left(\frac{\omega_{r} s}{c}\right) \\
& =\left[W_{0} e^{-\left(\omega_{r} s / 2 Q_{0} c\right)}\right] e^{-\left(\omega_{r} s / 2 Q_{\text {opics }} c\right)} \sin \left(\frac{\omega_{r} s}{c}\right),
\end{aligned}
$$

where $Q_{\text {ring }}$ is the $Q$ factor of the wake function due to the ion cloud along the whole ring and $Q_{\text {optics }}$ is due to the variation of beam size around the ring. We can also derive the following relationship from Eq. (16):

$$
\frac{1}{Q_{\text {ring }}}=\frac{1}{Q_{0}}+\frac{1}{Q_{\text {optics }}}
$$

In practice, the $Q_{\text {ring }}$ is found by numerically fitting the beam-ion wake function given by Eq. (8). For the wake function of each type of ion species shown in Fig. 10(a), we get $Q_{\text {ring }}=4.49$. $Q_{0}$ is due to the nonlinear space charge effect and has a value $Q_{0}=9$. Therefore, from the above equation, $Q_{\text {optics }}=8.9$. Note that the factor $Q_{\text {optics }}$ is about 
the same as $Q_{0}$. This finding agrees with the analysis above where the beam size variation effect and nonlinear space charge force contribute similar damping rates to the beamion instability. The factor $Q_{\text {optics }}$ represents the relative variation of the ion frequency due to beam size variation [Eq. (15)] and does not vary with ion species, as expected.

\section{NUMERICAL SIMULATIONS}

Time-domain simulations have a number of advantages in the study of beam-ion instabilities: the nonlinearity of the interaction force between electron beam and ion-cloud can be included, the effects of beam optics and bunchtrain gap with arbitrary beam fill pattern are readily visible, and a realistic vacuum model with multigas species is

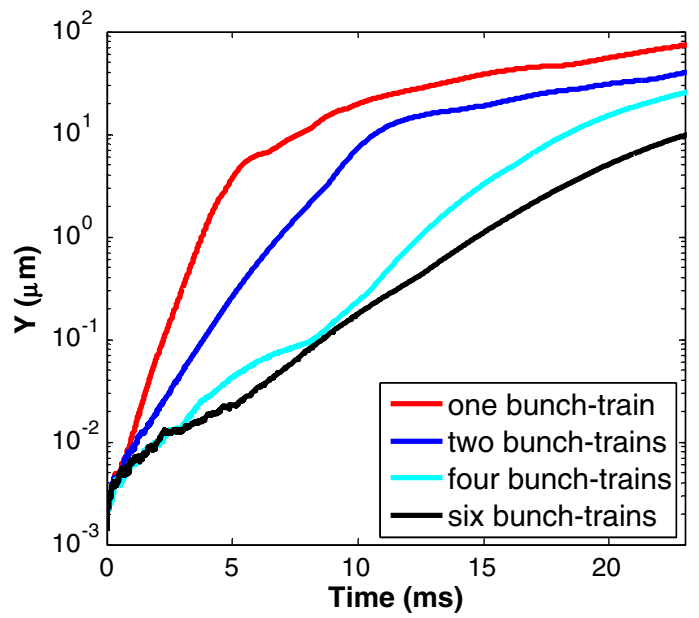

FIG. 17. Simulated beam-ion instability for different bunchtrain fill patterns. The total beam current is $500 \mathrm{~mA}$ with total bunch number of 280 in all cases. Zero chromaticity and total vacuum pressure of 0.37 nTorr are used in the simulation. Radiation damping not included. straightforward. A particle-in-cell code based on a weakstrong model is used here [10].

Figure 17 shows the simulated beam instability with different bunch-train fill patterns and total beam current of $500 \mathrm{~mA}$. The total vacuum pressure is $0.37 \mathrm{nTorr}$ for all the simulations in this section. The simulations show there is at first an exponential growth of the instability which is eventually slowed down by the nonlinearity of space charge forces when the amplitude is larger than the beam size. The time evolutions with four and six bunch train are very similar. The simulations agree well with measurements. The six-bunch-train fill pattern appears to be the optimum one with a growth time of $2.72 \mathrm{~ms}$. This agrees well with the analytical model in which the calculated growth time is $3.18 \mathrm{~ms}$. The exponential growth rate in the experiment is still unknown. However, it is known that a weak instability can appear with the six-bunch-train beam fill pattern which suggests a growth time slightly shorter than the radiation damping time of $5.3 \mathrm{~ms}$. The simulation is therefore within a factor of 2 of the measurements.

Figure 18 shows the time evolution of bunch-by-bunch vertical oscillation amplitudes with the six-bunch-train fill pattern. The overall amplitude grows exponentially in time after the initial onset of instability. The instability is still in an exponential regime after $20 \mathrm{~ms}$ due to its slow growth.

Simulation also allows us to study the nonlinear regime. Figure 19(a) shows the unstable behavior of a single bunch-train beam. Instead of mode number, the frequencies of modes are shown in order to compare with the frequencies of the impedance. In this case, the unstable modes all have frequencies below $50 \mathrm{MHz}$. In addition, three main peaks are clearly seen which agree with the multi-ion impedance calculations shown in Fig. 12. The unstable modes driven by $\mathrm{H}_{2}$ ions in the $125-150 \mathrm{MHz}$ region are very weak and hard to see. This again agrees
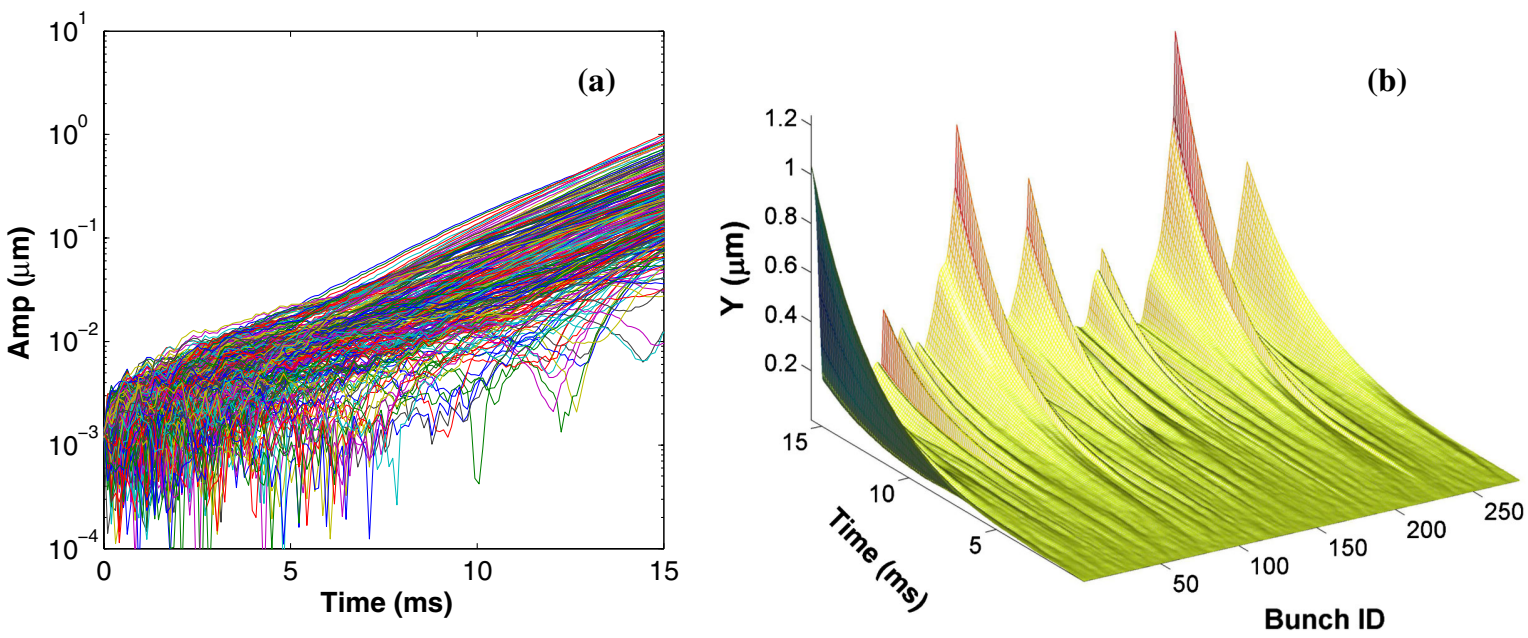

FIG. 18. Simulation of vertical beam-ion instability for the six-bunch-train fill pattern: (a) 2D plot and (b) 3D plot of vertical amplitude growth. The vertical oscillation amplitude in (a) is on a logarithmic scale, and in (b) is on a linear scale. The different lines in (a) represent each separate bunch. The total beam current is $500 \mathrm{~mA}$ and the total bunch number is 280 . 

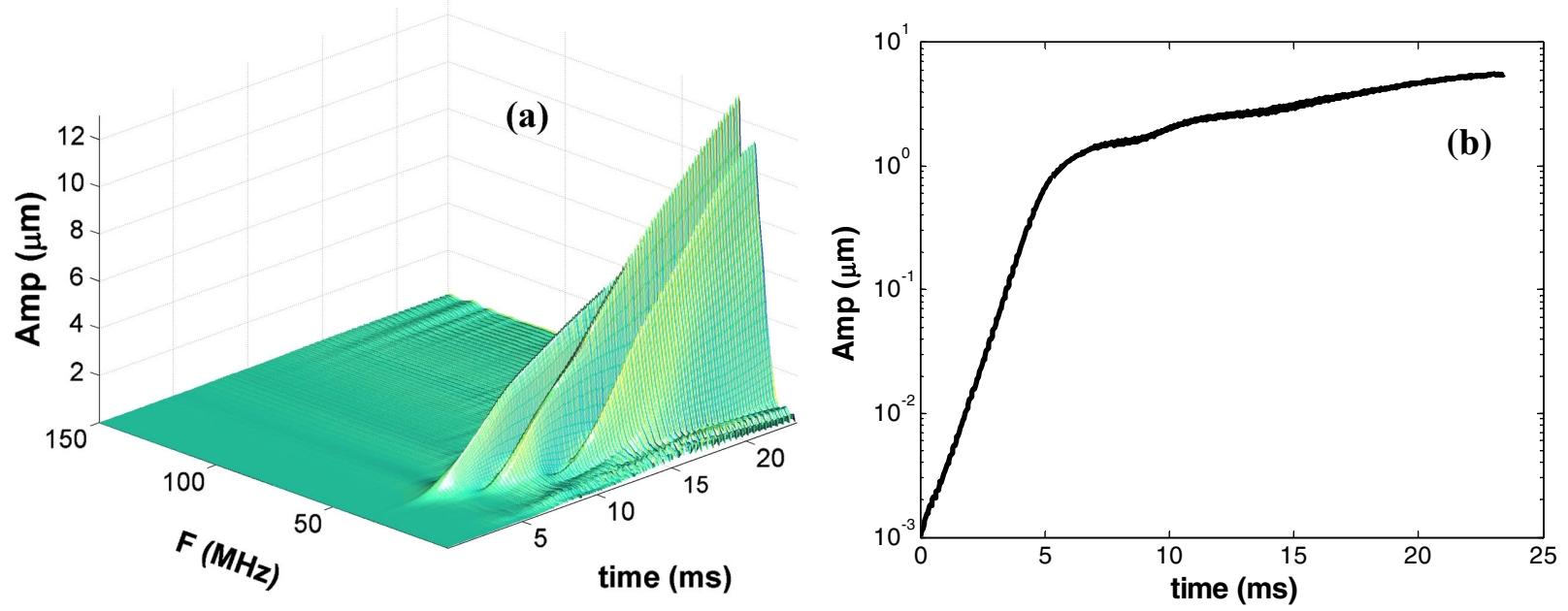

FIG. 19. (a) All unstable modes and (b) a particular unstable mode 336 for single bunch-train fill pattern with the same simulation parameters in Fig. 17. The frequencies of the unstable modes are shown in (a) for direct comparison with characteristic frequencies of the ion-cloud impedance. Radiation damping not included.

with theory and measurements, where hydrogen-driven sidebands are rarely seen. A particular mode 336 is highlighted in Fig. 19(b) with the vertical axis on a logarithmic scale. Both the linear and nonlinear growth regimes are visible. In this case, mode 336 has exponential growth when the amplitude is less than $10 \mu \mathrm{m}$ and thereafter saturates to the order of the beam size in agreement with measurements.

\section{CONCLUSIONS}

This paper summarizes comprehensive measurement, analysis, and simulation of beam-ion interactions in the vertical plane at SPEAR3. An instability was first observed under nominal vacuum conditions with a single bunch train. The characteristics of the beam-ion instability were confirmed by varying vacuum pressure and beam size. The instability causes the beam to oscillate with amplitude saturation of order of the vertical rms beam size. Similarly a blowup of the vertical beam size was observed with no bunch-train gap. The instability is also sensitive to fill pattern and chromaticity. Multibunch-train fill patterns were found to be helpful to suppress the instability. A six bunch train is the optimum fill pattern and the optimized bunch-train gap is about 1-2 ion oscillation wavelengths. At $500 \mathrm{~mA}$ beam current the beam-ion instability can be suppressed by slightly raising the chromaticity.

In this paper, we introduced an impedance model to study the beam-ion instability. The ion-cloud impedance is broadband by nature due to the variation of beam size around the storage ring and due to the multiple gas species in the vacuum chamber. The resulting impedance provides clear physical insight into the physics of beam-ion instabilities.

Analytical evaluations of multi-bunch-train beam-ion instabilities include beam optics, multiple gas species, nonlinear space charge forces, and realistic beam fill patterns. Each of these factors can provide damping under the correct operating conditions. Damping due to beam optics and nonlinear space charge forces are clearly seen in the analysis. Similarly the multiplicity of spikes in the beam spectrum is explained by the multiple gas-species model.

Overall we found excellent agreement between measurement, analysis, and simulation in many ways. For instance, the frequency of the measured beam spectrum agrees well with analytical calculations in the frequency domain and simulations in the time/frequency domain. The calculated and simulated growth rates of beam-ion instabilities with multibunch trains agrees with measurements, often within a factor of 2 even with the uncertainty of gas composition and the inability to measure real growth rates. With the recent purchase of transverse bunch-by-bunch feedback front-end electronics, measurements of beam-ion interactions in the time domain will be extended in SPEAR3.

\section{ACKNOWLEDGMENTS}

The authors would like to acknowledge the SPEAR3 operations team for invaluable support during the measurements and thank Rodney Pak for providing the vacuum data. We also thank D. Teytelman for providing the bunchby-bunch measurement data in the time domain. This work is supported by DOE contract No. DE-AC02-76SF00515.

[1] T. O. Raubenheimer and F. Zimmermann, Phys. Rev. E 52, 5487 (1995).

[2] J. Byrd, A. Chao, S. Heifets, M. Minty, T. Raubenheimer, J. Seeman, G. Stupakov, J. Thomson, and F. Zimmermann, Phys. Rev. Lett. 79, 79 (1997). 
[3] J. Y. Huang, M. Kwon, T.-Y. Lee, I. Ko, Y. Chin, and H. Fukuma, Phys. Rev. Lett. 81, 4388 (1998).

[4] M. Kwon, J. Huang, T.-Y. Lee, I. Ko, Y. Chin, H. Fukuma, M. Isawa, K. Ohmi, and M. Tobiyama, Phys. Rev. E 57, 6016 (1998).

[5] H.S. Kang et al., in Proceedings of the 10th European Particle Accelerator Conference, Edinburgh, Scotland, 2006 (EPS-AG, Edinburgh, Scotland, 2006), p. 2771.

[6] R. Nagaoka et al., in Proceedings of the 2007 Particle Accelerator Conference, Albuquerque, New Mexico (IEEE, New York, 2007), p. 2019.
[7] B. Jiang, G. Xia, L. Han, G. Liu, Z. Dai, and Z. Zhao, Nucl. Instrum. Methods Phys. Res., Sect. A 614, 331 (2010).

[8] G. V. Stupakov, KEK Proceedings 96-6 (KEK, Tsukuba, Japan, 1996), p. 243.

[9] E. S. Kim and K. Ohmi, Jpn. J. Appl. Phys. 48, 086501 (2009).

[10] L. Wang, Y. Cai, T. O. Raubenheimer, and H. Fukuma, Phys. Rev. ST Accel. Beams 14, 084401 (2011).

[11] L. Wang, M. Pivi, T. O. Raubenheimer, and J. Safranek, Report No. SLAC-PUB-15353, 2013.

[12] A. Chao, Physics of Collective Beam Instabilities in High Energy Accelerators (Wiley, New York, 1993). 